\title{
MANORIAL AND GRANGE COMPLEXES AS A CULTURAL HERITAGE OF THE AGRICULTURAL LANDSCAPE IN THE MANAGEMENT OF COMMUNE SPACE: A CASE STUDY OF TARNOWO PODGÓRNE, WIELKOPOLSKA
}

\author{
AgnieszKa Wilkaniec ${ }^{1}$, Magdalena SZCZEPAŃSKA ${ }^{2}$ \\ ${ }^{1}$ Faculty of Horticulture and Landscape Architecture, University of Life Sciences, Poznań, Poland \\ ${ }^{2}$ Institute of Socio-Economic Geography and Spatial Management, Adam Mickiewicz University in Poznań, \\ Poland
}

Manuscript received: December 20, 2016

Revised version: February 20, 2017

\begin{abstract}
WiLKANIEC A., SZCZEPAŃSKA M., 2017. Manorial and grange complexes as a cultural heritage of the agricultural landscape in the management of commune space: A case study of Tarnowo Podgórne, Wielkopolska. Quaestiones Geographicae 36(2), Bogucki Wydawnictwo Naukowe, Poznań, pp. 107-123, 1 table, 9 figs.

AвSTRACT: Manorial and grange complexes are characteristic elements of the rural cultural landscape; they constitute significant evidence of the farming tradition of a region. Unfortunately, nowadays these complexes have often become dilapidated, their legibility has been obliterated and there have been radical changes in the spatial context. The aim of this study was to determine whether manorial and grange complexes were given proper attention in the development and strategic plans worked out by communes. The commune of Tarnowo Podgórne, stretching along the western boundary of Poznań city, was selected as a case study and research area. An attempt is also made to recognise what protective provisions the local law makes for those complexes. The authors think that the quality of plans concerning the facilities under study is unsatisfactory. The Land Use Plan includes recommendations to protect nonexistent facilities and lists a complex located beyond the commune boundaries. Although the Plan emphasises the significance of manorial and grange complexes, there are no local spatial development plans for most of them. Some plans referring to the spatial structures under analysis are imprecise and incomplete, which results in ineffective protection.
\end{abstract}

KEY WORDS: land use plan, local spatial development plans, manorial and grange complexes, rural landscape, spatial management, Tarnowo Podgórne, Wielkopolska

Agnieszka Wilkaniec, Faculty of Horticulture and Landscape Architecture, Poznań University of Life Sciences, Dąbrowskiego 159, 60-594 Poznań, Poland; e-mail:ktzagawi@up.poznan.pl

Magdalena Szczepańska, Institute of Socio-Economic Geography and Spatial Management, Adam Mickiewicz University,

Krygowskiego 10, 61-680 Poznań, Poland; e-mail: szmagda@amu.edu.pl

\section{Introduction}

The agricultural landscape is perceived as a section of the Earth's surface whose main function is agriculture. There are four approaches to the phenomena and processes occurring in it, i.e. ecological, geographical, techno-economic and spatial planning. The geographical approach treats the agricultural landscape as a working spatial arrangement which consists of both, elements of nature (soils, water, terrain, etc.) and socio-economic elements, such as the land-use pattern, the agrarian structure, the type of development, and others (Kostrowicki 1975; Cymerman et al. 
1992). Therefore, manorial and grange complexes are characteristic elements of the rural cultural landscape of Wielkopolska, including areas surrounding Poznan, and they constitute significant evidence of the farming tradition of the region. Being seats of land estates or accessory granges, they were regularly dispersed at a distance of a few kilometres. They were specific elements in the spatial arrangements of villages, or they stood as self-contained facilities. Both their spatial structure and the buildings forming a complex were often of high artistic and significant historical value. Those values could be seen not only in palace or manorial and park complexes, but also in grange complexes (Jakimowicz 1994). It is noteworthy that in the past a grange and its farming areas were particularly important elements of a complex. The profit they generated enabled the maintenance of the manorial part. Unfortunately, nowadays these complexes have often become dilapidated, their legibility obliterated, and there have been radical changes in the spatial context (Chojnacka, Wilkaniec 2009; Rzeszotarska-Pałka 2016; Szczepańska, Wilkaniec 2016). In spite of their partial destruction, manorial and grange complexes are important elements of the rural cultural landscape. Green spaces connected with former parks, roadside alleys planted by former landowners, as well as manorial and grange buildings are often landmarks and significant elements of scenic interiors (NiedźwiedzkaFilipiak 2009). In many cases functional links between manorial and grange complexes and the contemporary agricultural landscape have been broken. Grange buildings are no longer used for the purposes of agricultural production. As a result of privatisation, grange complexes have now different owners than the surrounding farmland. In the suburban zone farmland is being replaced by buildings. In consequence, relics of manorial and grange complexes are physically separated from the agricultural landscape, which decreases their significance in it. In the past they were particularly important components of the landscape, and together with roadside alleys and the grange land pattern influenced not only the character of the habitat space, but also the open landscape and layout of fields. What shows the significance of those complexes for the landscape is one of the latest landscape typologies: Chmielewski et al. (2015) distinguish subtype 8e, "large palace-park and monastic complexes as well as other compositions of architecture, green spaces and water bodies". The historical and contemporary significance of those components for the landscape is also noted by other authors: Rzeszotarska-Pałka (2006), Gubańska (2008), Napierała (2009), Rylke, Gąsowska (2009), Raszeja (2010), Kijowski et al. (2011), Kowalski (2013). They can be preserved and protected with tools used in spatial planning, as is done in many European countries where various elements of the cultural heritage are preserved and protected (Daugstad et al. 2006; Swensen, Jerpasen 2008). In Poland, local spatial development plans are considered to be the most effective tools (Böhm 2006; Raszeja 2002; Raszeja et al. 2010). Jaszczuk-Skolimowska (2008) emphasises the significance of spatial planning for the proper development of rural spatial structures. It is possible to adapt historical spatial development models to contemporary needs by means of spatial planning. However, MygaPiątek (2007) observes that problems of the cultural landscape are not sufficiently represented in spatial planning despite the possibility to use tools available in the Polish legal system. Many authors present spatial planning as one of the basic tools for space and landscape development, especially in suburban areas, which are particularly endangered by rapid changes (Dubel 2003, Żarska 2003). Szyda (2013) writes about the urbanisation of rural areas in the impact zone of a city (with reference to Radom, Częstochowa and Kielce) and about the use of planning tools in this zone by local governments. The author points to the relation between the scale of urbanisation (as measured by selected indices) and the coverage of the commune area by local plans. The most urbanised rural communes (usually located nearest to the city limits) had the greatest coverage in local spatial development plans. It may indicate that commune authorities are increasingly aware of the significance of planning tools for the spatial policy, but it may also indicate a high building pressure exerted on those areas. In consequence, there are more local spatial development plans prepared for areas to be built up. A study by Feltynowski (2013) also shows that in the rural communes bordering on Łódź the area covered by local spatial development plans is great.

The contemporary rural landscape was particularly influenced by the spatial changes in the 


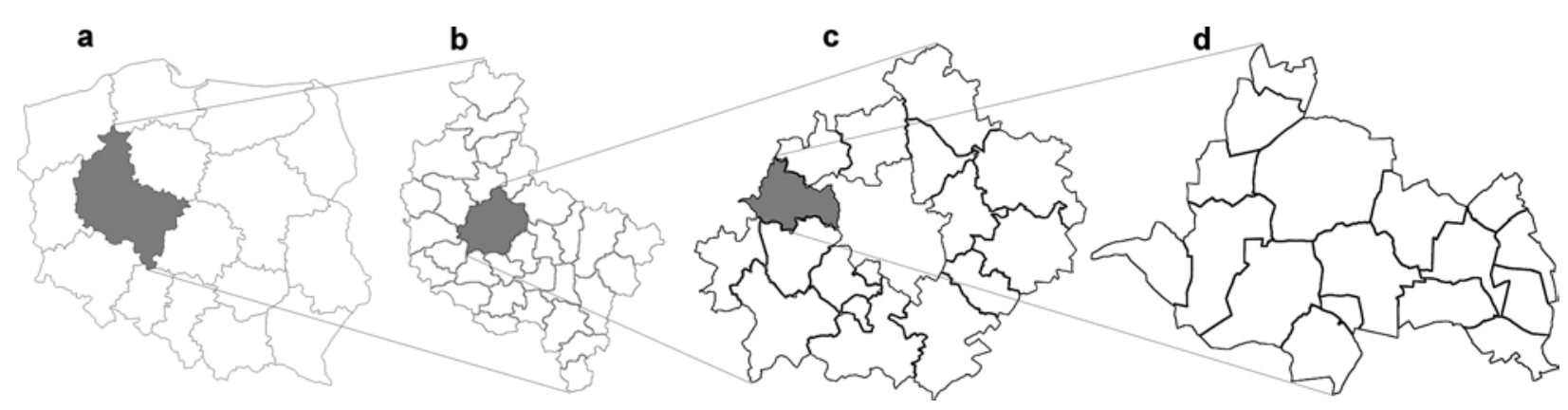

Fig. 1. Tarnowo Podgórne commune in the administrative division of Poland: (a) Poland/ voivodeships; (b) Wielkopolska/ poviats; (c) Poznań poviat/ communes; (d) Tarnowo Podgórne commune/ villages (sołectwa). Source: own compilation.

agrarian structure which took place in the 1990s. The transformation of the ownership and size structure of farms was intended to make them meet the requirements of a market economy with simultaneous orientation to multifunctional rural development (Głębocki 1998). Jedut (1998) notes the significance of restructuring implemented by the Agricultural Property Agency of the State Treasury in the protection and management of historical buildings, including manorial and grange complexes taken over from state farms, plant and animal breeding enterprises, and the National Land Fund.

The aim of this study is to determine whether manorial and grange complexes, which are valuable elements of the rural cultural landscape, were given proper attention in the development and strategic plans worked out by communes. Also examined are protective measures that the local law provides for them. The article analyses the local law in detail, verifies its enforcement, the state of preservation of selected complexes, and their operation, today and in the future.

\section{Research area and methods}

The commune of Tarnowo Podgórne, located west of the limits of Poznań, was selected as the case study and research area. According to Kondracki's (2000) psycho-geographical division of Poland, the commune of Tarnowo Podgórne lies in the sub-province of the Southern Baltic Lake District (314-316), in the macroregion of the Wielkopolska Lake District (315.5), and the mesoregion of the Poznań Lake District (315.51). According to Krygowski's (1961) geomorphological division of the Wielkopolska Lowland, the commune is located in the region of the Poznan
Upland (VIII), which consists of the following subregions: the Międzyrzecz-Pniewy Hills (VII1), the Poznań Plain (VIII6), and the Szamotuły Plain (VIII7). The agricultural landscape is rather monotonous due to minimal diversification of the terrain and its cover (farmland with a high share of large fields, a low woodiness rate, and advancing urbanisation). Besides a few natural and scenic values (the Sama River valley, Lake Lusowo), the complexes under study are significant elements diversifying the landscape.

The commune of Tarnowo Podgórne is located in Wielkopolska voivodeship, Poznań poviat, in the western suburban zone of the city of Poznań ${ }^{1}$ (Fig. 1).

In the recent years it has been one of the most significant locations for the development of residential functions, services, production and storage. Formerly the commune was predominantly agricultural. At present we can observe its very rapid development, which considerably limits the use of land for agricultural purposes and causes changes in historical spatial layouts. The development of those functions is stimulated by the location of the commune in the direct neighbourhood of the city of Poznan and a convenient road network (roads 92, 184, 307 and S-11) in the

In Poland the voivodeship is the highest degree unit of administrative division, a local government unit and a government administration unit. At present there are 16 voivodeships, which consist of poviats (a second-degree local government unit and a unit of the administrative division). The commune is the lowest-degree unit of the administrative division. The solectwo is an auxiliary unit in a commune. Its territory comprises a fragment, one or a few villages, hamlets or settlements. Regional spatial planning is related with the voivodeship, whereas local spatial planning is related with the commune. 


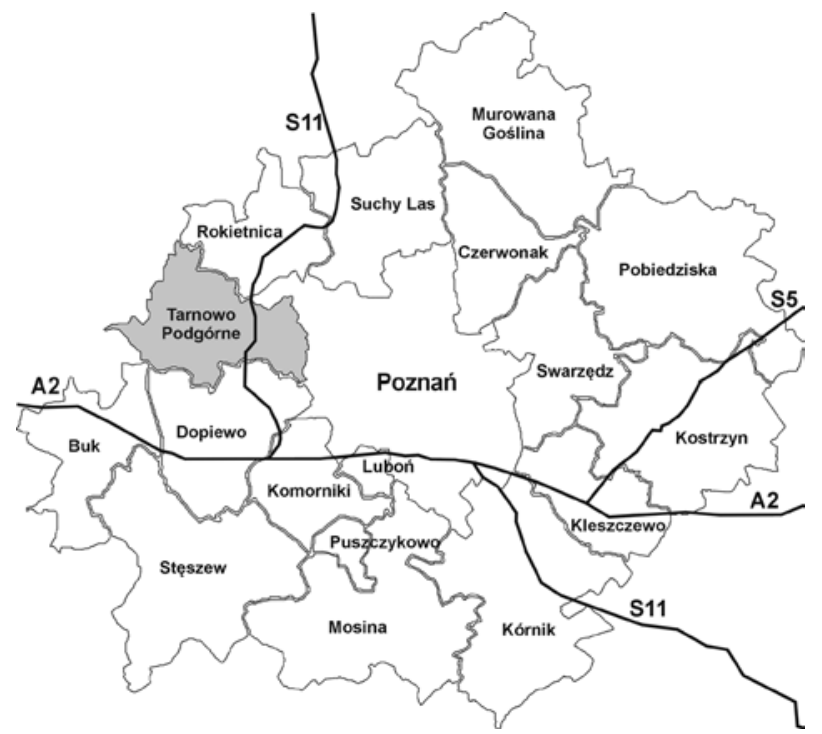

Fig. 2. Location of Tarnowo Podgórne commune in the suburban zone of the city of Poznan against the administrative division and the road network. Source: own compilation.

vicinity of the A2 motorway. The commune has also good public transport connections (Fig. 2).

Good-quality soils and the closeness of Poznan city, which on the one hand creates a demand for agricultural products, but on the other hand provides alternative, non-agricultural employment for commune inhabitants, have enabled relatively good development of agriculture in the commune. These opportunities, combined with the inhabitants' thrift, have resulted in the development of two models of farming in the commune: market agriculture and auxiliary agriculture. Their coexistence and other functions show the multifunctional development of rural areas.

The farmland area in the commune is 7,492 ha, i.e. $73 \%$ of the total area. However, this share keeps decreasing. Arable land makes up 92\% of all farmland (6,870 ha), orchards - 139 ha, permanent grassland - 365 ha, and permanent pastures - 118 ha. The development of agriculture in the commune may be potentially aided by a high share of good-quality soils $(21.8 \%$ - soils of classes I-III, $46.2 \%$ - class IV soils). However, due to periodical droughts which make regular and optimal yields impossible, and due to the location of the commune in the urban agglomeration of Poznań, more and more farmland is used for other purposes than agriculture. Usually it is used for building or business purposes. The average area of a farm in Tarnowo Podgórne commune is $9.12 \mathrm{ha}$, which is much smaller than the average farm area in the voivodeship. A large number of farmers also run non-agricultural businesses. The farms that derive an income only from agriculture are larger than 10 ha. They constitute the greatest proportion of farms in the commune (Tarnowo Podgórne Land Use Plan, 2011).

Manorial and grange complexes are significant and characteristic elements of the commune's landscape and one of the major values of its cultural heritage. The research included a detailed analysis of its Land Use Plan and determined the coverage of the complexes by local spatial development plans. It also analysed provisions in the plans concerning the preservation, protection and development of the complexes and their surroundings. It was determined whether manorial and grange complexes were included in other documents issued by the commune (revitalisation programmes, development plans, etc.). The focus was on the complexes listed in the Land Use Plan. The state of preservation of their individual elements, i.e. residential buildings (e.g. palaces, manors, steward's houses), accompanying parks, gardens and grange yards, was evaluated according to a graded scale, developed by the present authors and already used many times to assess the state of preservation of buildings and structures which are elements of the cultural landscape (Chojnacka, Wilkaniec 2009; Szczepańska, Wilkaniec 2016). The state of preservation was rated as follows: 5 - good, 4 - good, transformed, 3 transformed, neglected, 2 - transformed, degraded, 1 - traces preserved, and 0 - not preserved. Also analysed were the following factors: the location in the spatial structure of a settlement unit, the pattern of development and use of neighbouring areas, the current function, and ownership relations. It was also determined whether individual facilities or entire complexes had a conservation zone or were registered as monuments. Revitalising operations planned by the commune authorities were also identified. The information gathered in the study as well as the spatial and landscape analyses made it possible to indicate how those facilities could be protected.

\section{Manorial and grange complexes in the Land Use Plan}

In the $19^{\text {th }}$ and $20^{\text {th }}$ centuries there were about 20 manorial and grange complexes as well as 
granges in the present-day commune of Tarnowo Podgórne (Figs 3 and 4). About a dozen facilities or so still exist. They are at different states of preservation, ranging from a good state and full legibility of spatial elements to a state of preservation in the form of relics. Regulations in the Land Use Plan refer to 15 facilities (Table 1) - all of them were analysed in this study. The document mentions the possibility to develop different forms of tourism and recreation based on cultural heritage resources, such as manor parks (Tarnowo Podgórne Land Use Plan 2011, Part. A, p. 65). The Land Use Plan includes regulations concerning the protection of tree stands in cemeteries, parks, roadside alleys (where specific items are listed), recommendations to emphasise the existing scenic values in the agricultural landscape and to inventory the existing trees in parks and on roadsides (Tarnowo Podgórne Land Use Plan 2011, Part B, p. 34). Fragments of the Land Use Plan concerning

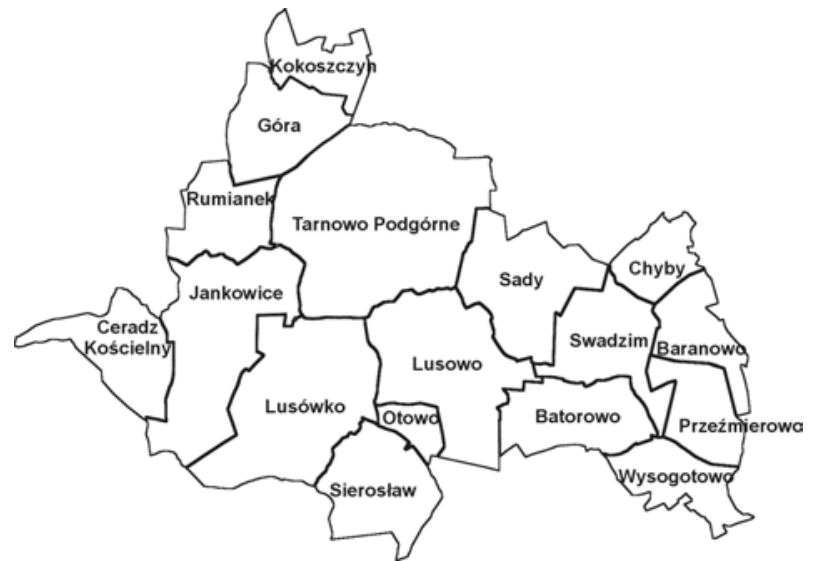

Fig. 3. Tarnowo Podgórne commune as the research area - division into solectwa (village units).

Source: own compilation.

the protection of the cultural heritage and monuments include recommendations to maintain historical facilities and their surroundings in an appropriate state. They provide rules concerning

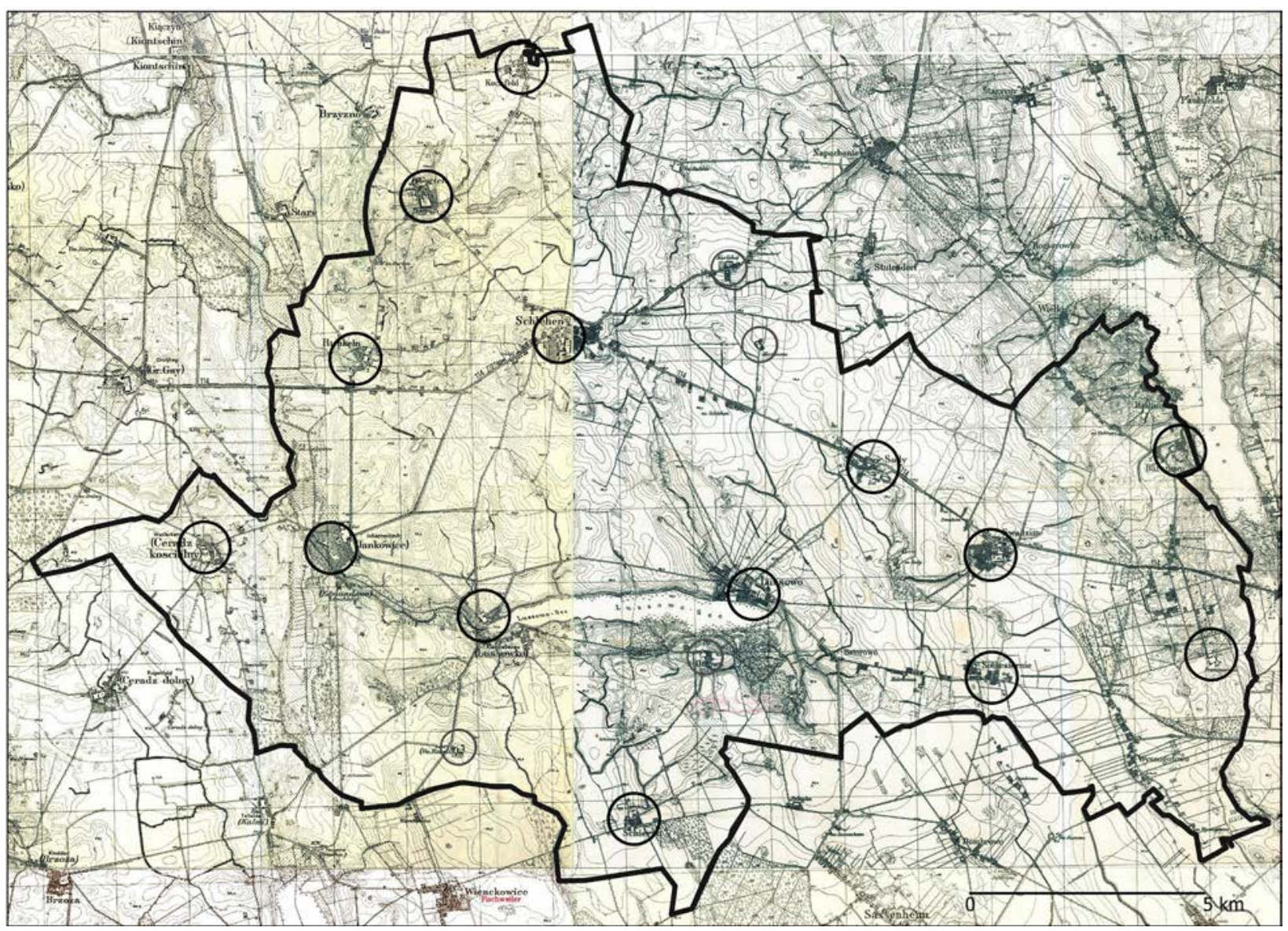

Fig. 4. Historical topographic map of the present-day Tarnowo Podgórne commune, published in the 1940s. Large circles mark the manorial and grange complexes listed in the Land Use Plan. Small circles mark the complexes not listed in the Plan.

Source: own compilation based on http://mapy.amzp.pl. 


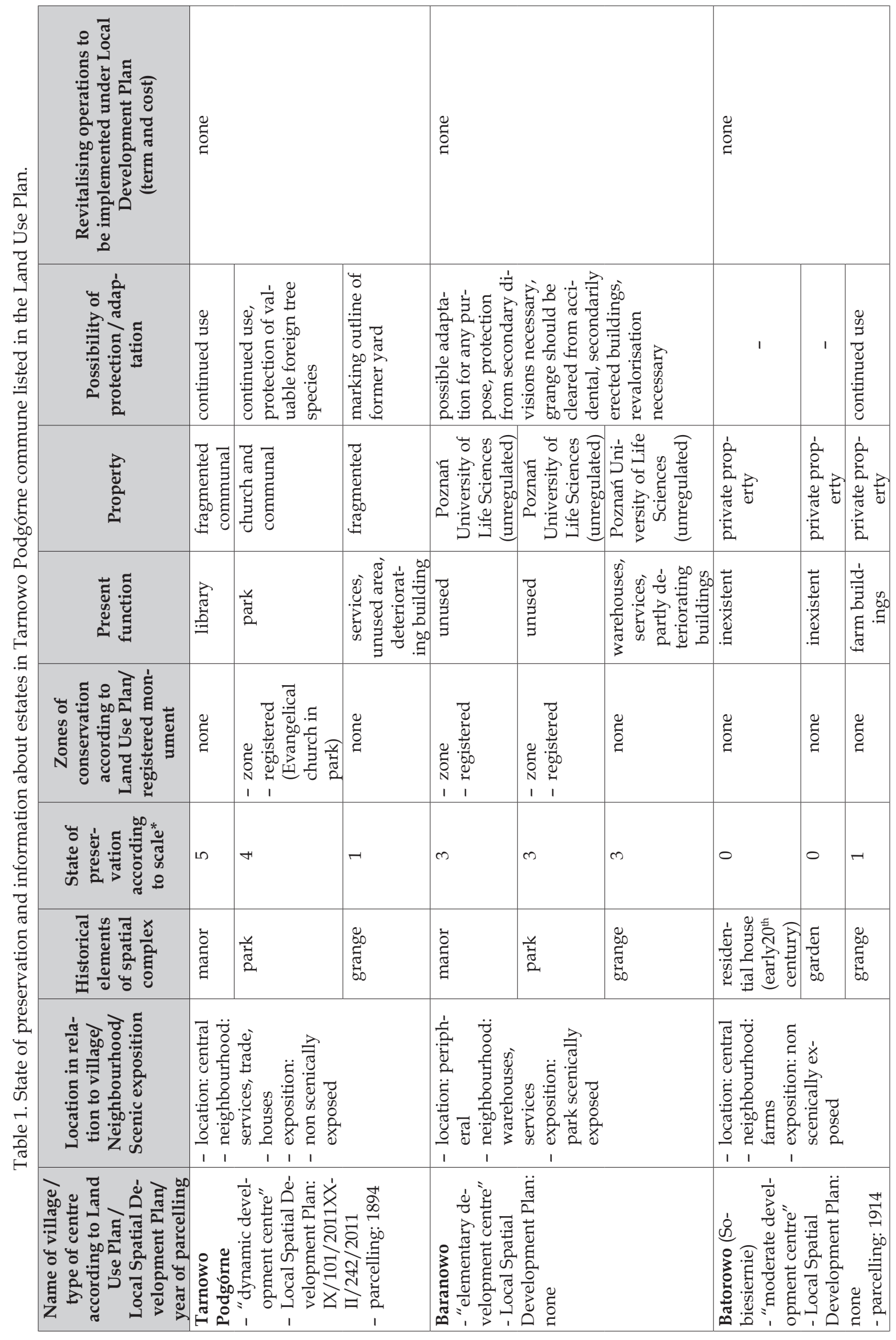




\begin{tabular}{|c|c|c|c|c|c|c|c|}
\hline ๕̊ & & $\begin{array}{l}\tilde{\Xi} \\
\tilde{\Xi}\end{array}$ & & & 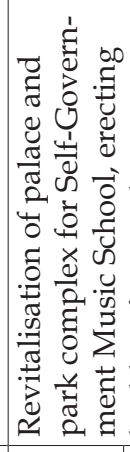 & 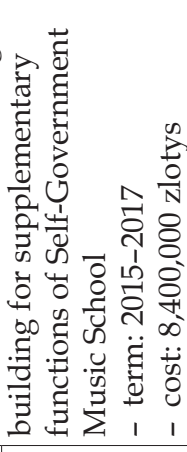 & 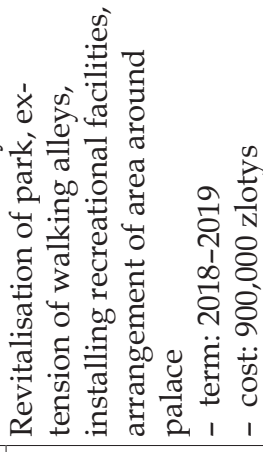 \\
\hline 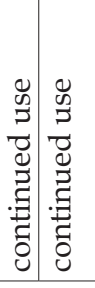 & 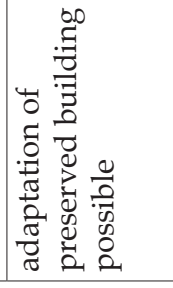 & 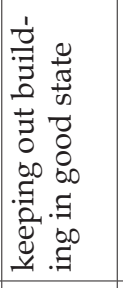 & 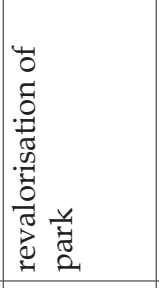 & 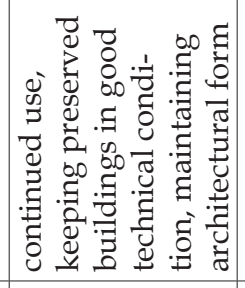 & 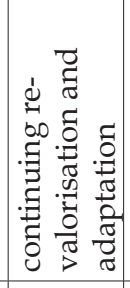 & 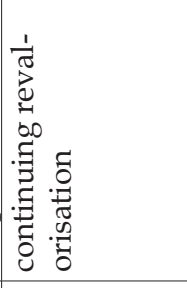 & 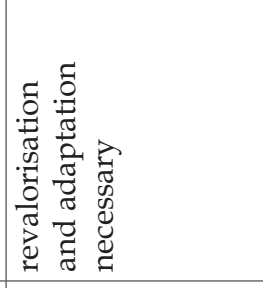 \\
\hline & & 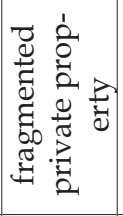 & 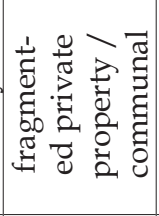 & 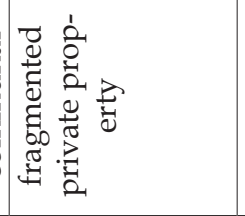 & 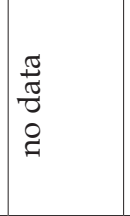 & 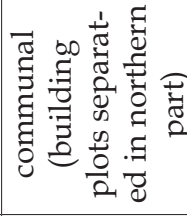 & 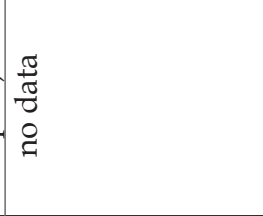 \\
\hline 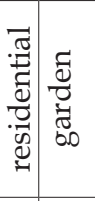 & 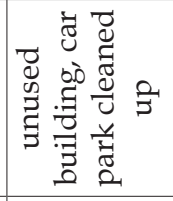 & 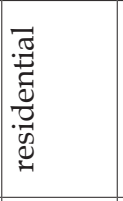 & $\begin{array}{l}\widetilde{J} \\
\mathbb{w} \\
\vec{\Xi} \\
\Xi\end{array}$ & 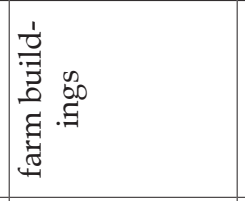 & 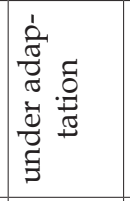 & $\begin{array}{l}\text { 䒕 } \\
\text { वू. }\end{array}$ & $\begin{array}{l}\tilde{\Xi} \\
\tilde{W} \\
\tilde{z} \\
\tilde{z}\end{array}$ \\
\hline 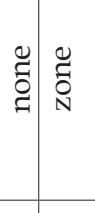 & 气̆ & $\begin{array}{l}\tilde{\Xi} \\
\text { ๕̊ }\end{array}$ & 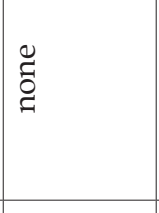 & $\begin{array}{l}\tilde{\Xi} \\
\text { こ }\end{array}$ & 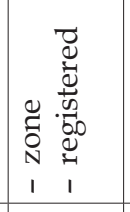 & 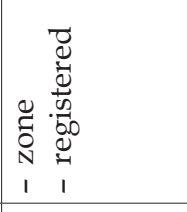 & 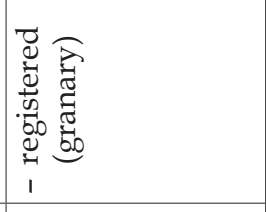 \\
\hline L) 10 & $r$ & $\neg$ & 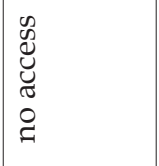 & $N$ & H & n & $r$ \\
\hline 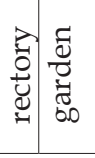 & 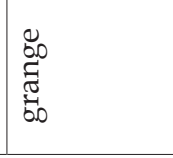 & 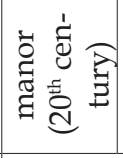 & 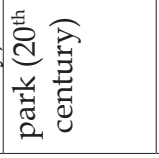 & 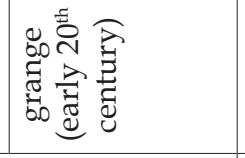 & $\begin{array}{l}\frac{\tilde{U}}{\pi} \\
\frac{\pi}{\pi} \\
2\end{array}$ & 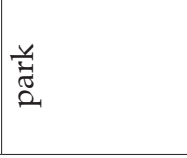 & $\begin{array}{l}0 \\
\infty \\
\tilde{\pi} \\
\tilde{d} \\
0\end{array}$ \\
\hline 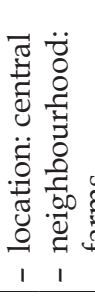 & 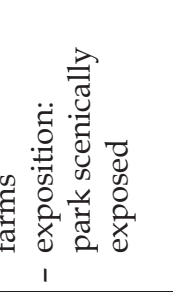 & 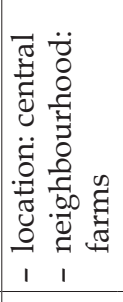 & 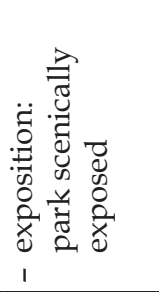 & & 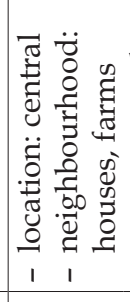 & 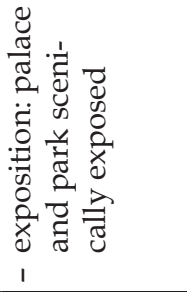 & \\
\hline 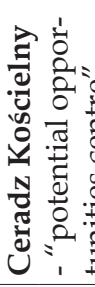 & 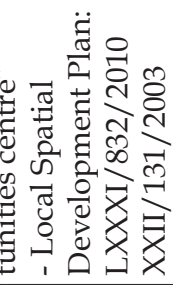 & 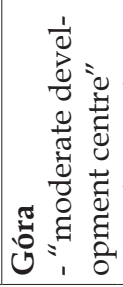 & 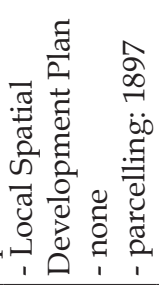 & & 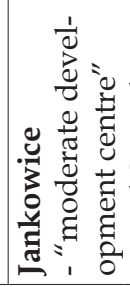 & 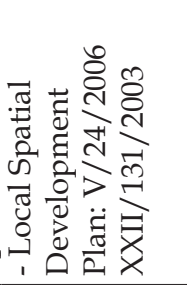 & \\
\hline
\end{tabular}




\begin{tabular}{|c|c|c|c|c|c|c|c|c|c|c|}
\hline 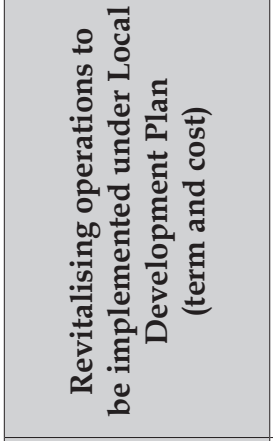 & \multicolumn{4}{|l|}{ ॄี } & \multicolumn{3}{|l|}{ 气્̃ } & \multicolumn{3}{|c|}{ 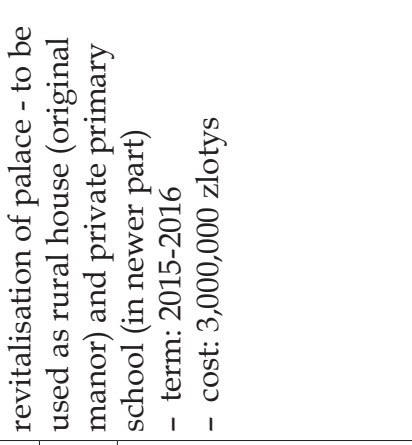 } \\
\hline 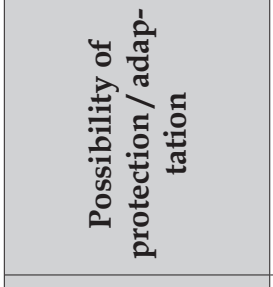 & & & 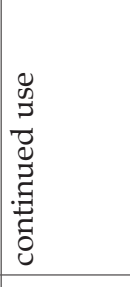 & 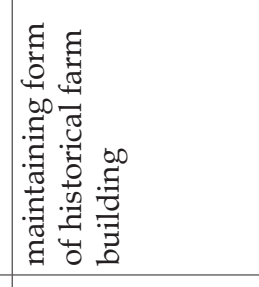 & 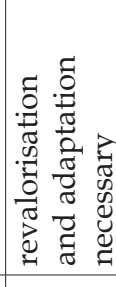 & 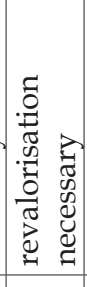 & 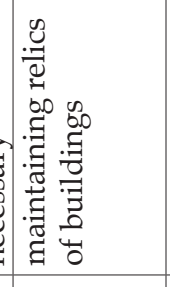 & 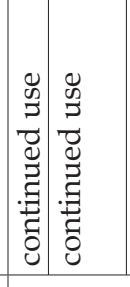 & & 1 \\
\hline $\begin{array}{l}\sum_{0}^{2} \\
\text { à } \\
\text { a }\end{array}$ & & & 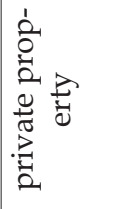 & 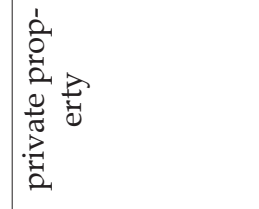 & 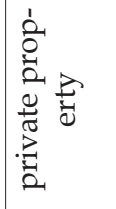 & 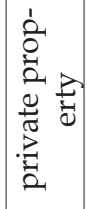 & 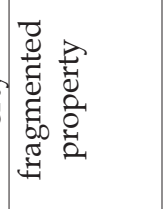 & \multicolumn{3}{|l|}{ 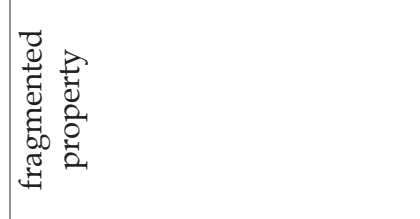 } \\
\hline 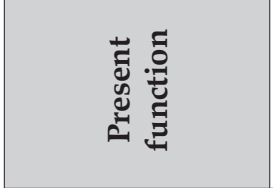 & & & 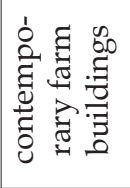 & 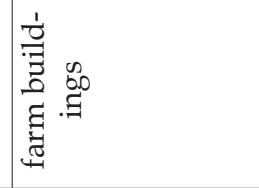 & $\begin{array}{l}\vec{Z} \\
\mathbb{W} \\
\stackrel{0}{\Xi} \\
\Xi\end{array}$ & $\begin{array}{l}\vec{\Xi} \\
\mathbb{u} \\
\vec{w} \\
\vec{g}\end{array}$ & 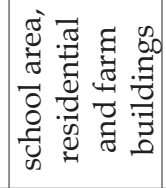 & 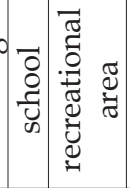 & & 1 \\
\hline 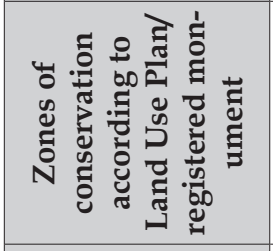 & 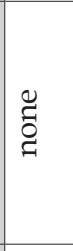 & 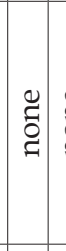 & 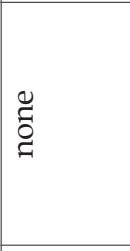 & $\begin{array}{l}\tilde{\Xi} \\
\check{\Xi}\end{array}$ & 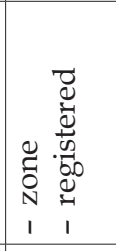 & 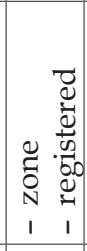 & 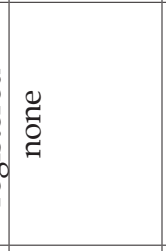 & 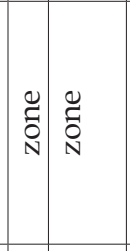 & \multicolumn{2}{|l|}{ §̊̆ } \\
\hline 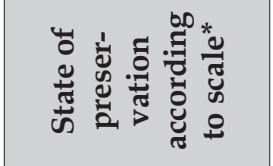 & 0 & 0. & - & $\neg$ & $\infty$ & $m$ & -1 & \begin{tabular}{l|l}
$|l|$ & 4
\end{tabular} & \multicolumn{2}{|l|}{0} \\
\hline 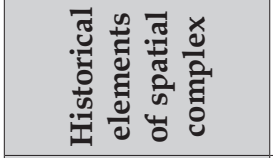 & 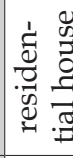 & & $\begin{array}{l}\infty \\
\infty \\
\tilde{\Xi} \\
\dot{\omega} \\
\end{array}$ & 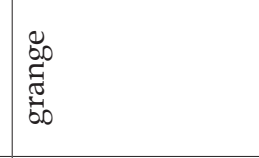 & $\begin{array}{l}\stackrel{\tilde{\Xi}}{\tilde{J}} \\
\frac{\pi}{2} \\
\stackrel{2}{2}\end{array}$ & $\begin{array}{l}\text { 节 } \\
\text { ટू }\end{array}$ & 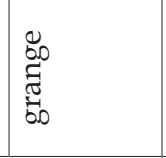 & 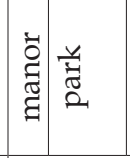 & \multicolumn{2}{|l|}{$\begin{array}{l}\tilde{\infty} \\
\infty \\
\tilde{J} \\
\tilde{\omega} \\
0.0\end{array}$} \\
\hline 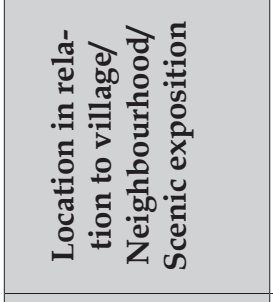 & 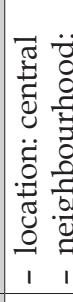 & & 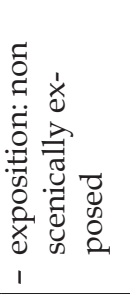 & 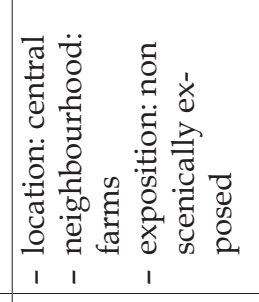 & \multicolumn{3}{|c|}{ 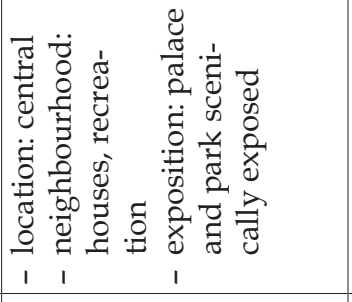 } & \multicolumn{3}{|c|}{ 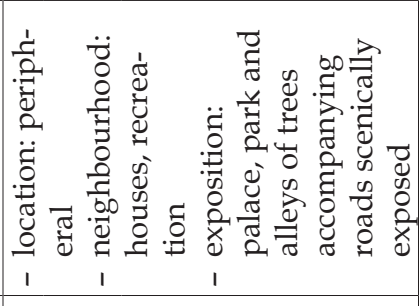 } \\
\hline 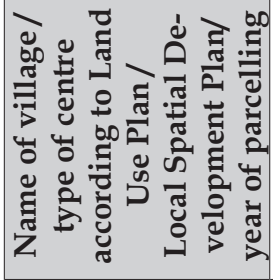 & 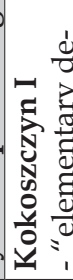 & & 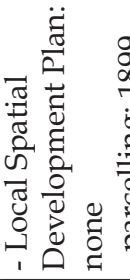 & 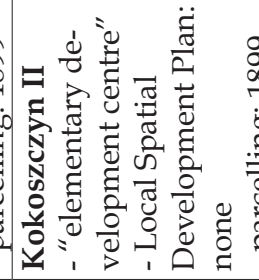 & 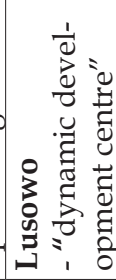 & 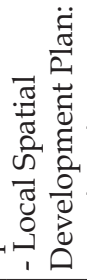 & 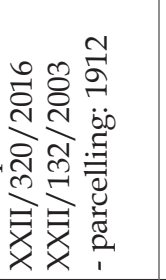 & 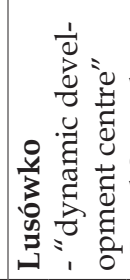 & 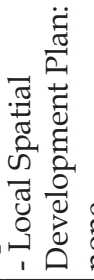 & \\
\hline
\end{tabular}




\begin{tabular}{|c|c|c|c|c|c|c|c|c|c|c|c|}
\hline \multirow{2}{*}{ 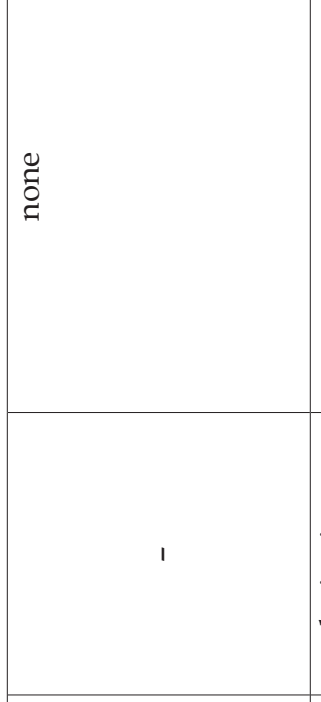 } & \multicolumn{3}{|l|}{ 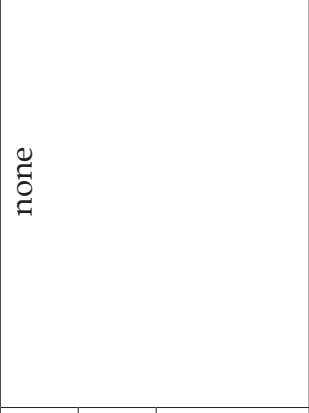 } & \multirow{2}{*}{ 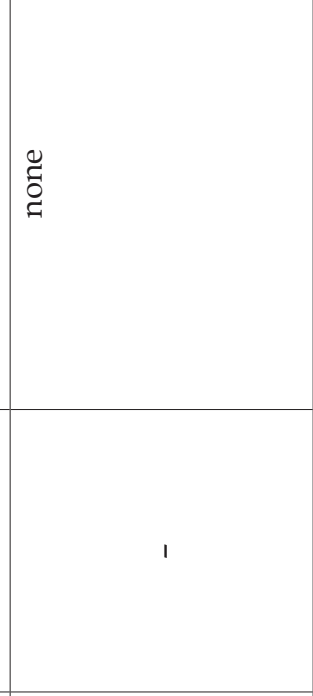 } & \multicolumn{3}{|c|}{ 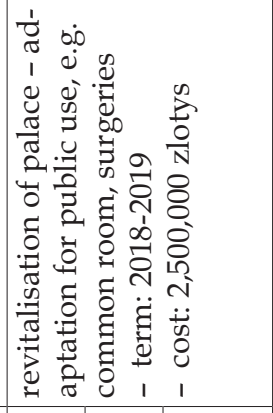 } & \multicolumn{3}{|l|}{$\begin{array}{l}\tilde{\Xi} \\
\check{\Xi}\end{array}$} & 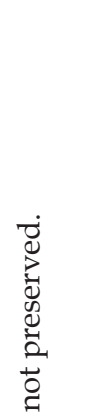 \\
\hline & \multicolumn{3}{|c|}{ 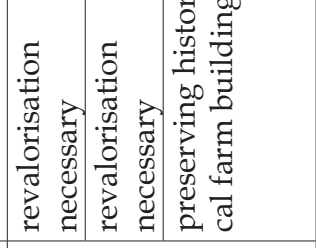 } & & 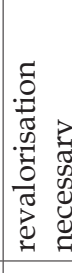 & 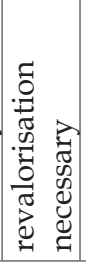 & 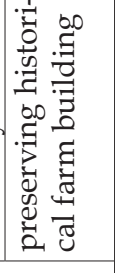 & 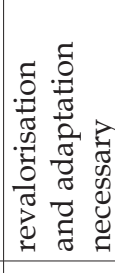 & 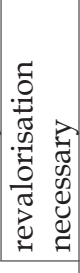 & 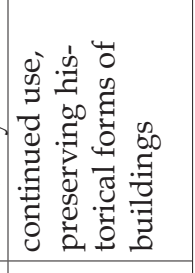 & 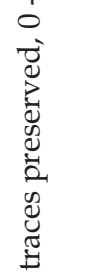 \\
\hline 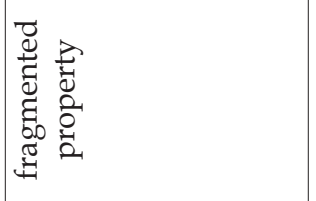 & \multicolumn{3}{|c|}{ 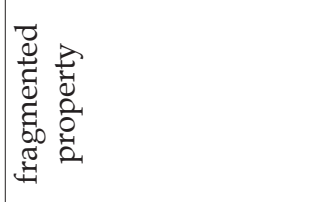 } & 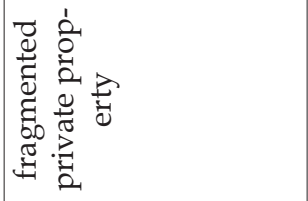 & \multicolumn{3}{|c|}{ 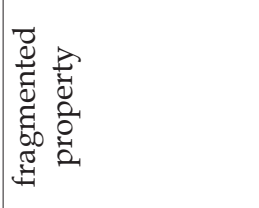 } & \multicolumn{2}{|l|}{ 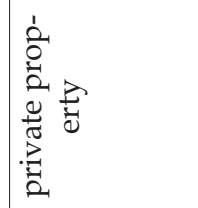 } & 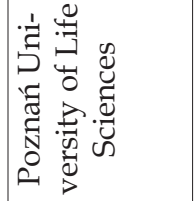 & 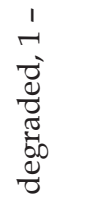 \\
\hline 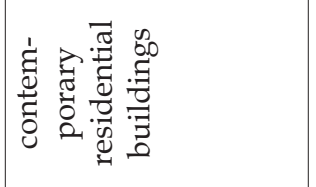 & 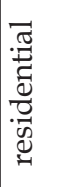 & 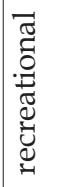 & 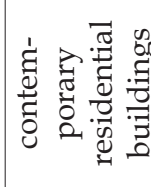 & 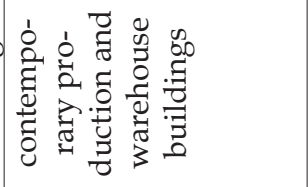 & 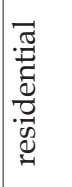 & 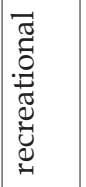 & 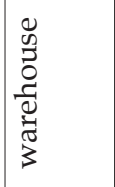 & 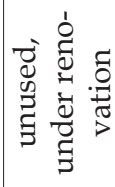 & 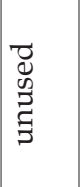 & 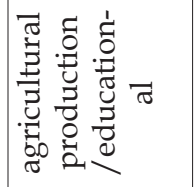 & 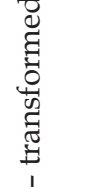 \\
\hline 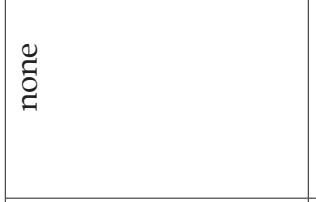 & ॄี & 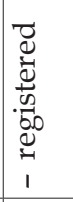 & 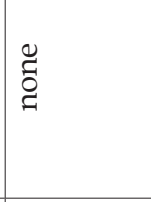 & 芯 & $\begin{array}{l}0 \\
\text { İ } \\
\text { N } \\
1\end{array}$ & 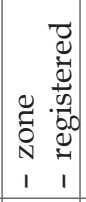 & ‡ี & 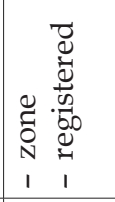 & 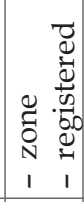 & : & 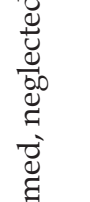 \\
\hline$\neg$ & $N$ & $\infty$ & $r$ & 0 & $\sim$ & $m$ & $r$ & $\infty$ & $\infty$ & $r$ & \\
\hline 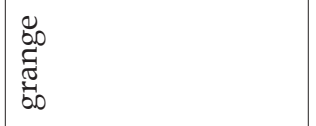 & 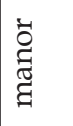 & 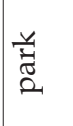 & 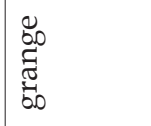 & 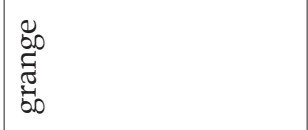 & 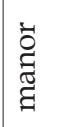 & 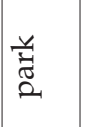 & 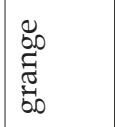 & 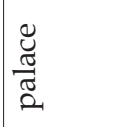 & $\begin{array}{l}\text { 弟 } \\
\text { ă }\end{array}$ & 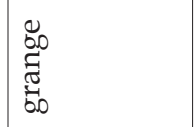 & 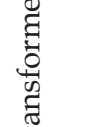 \\
\hline 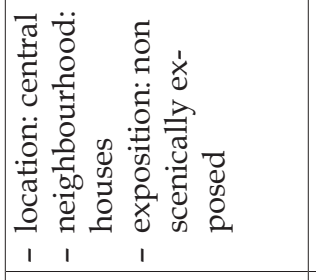 & 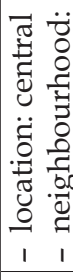 & 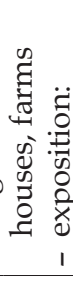 & 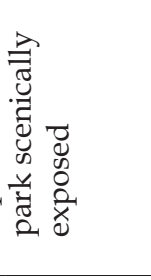 & 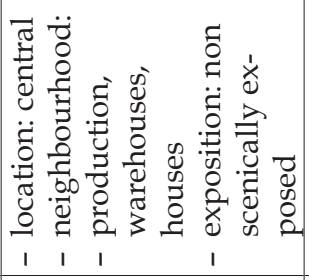 & 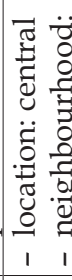 & 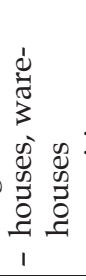 & 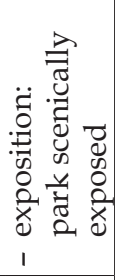 & 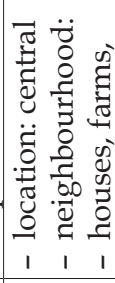 & 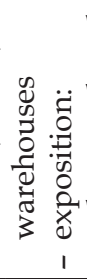 & 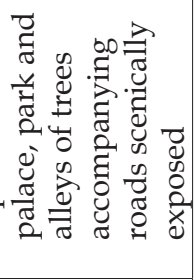 & 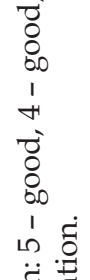 \\
\hline 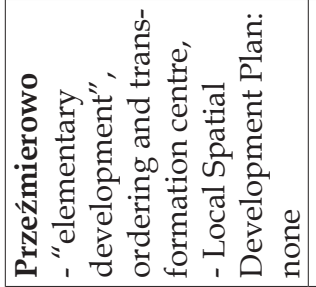 & 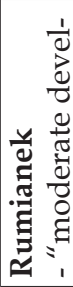 & 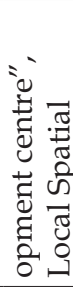 & 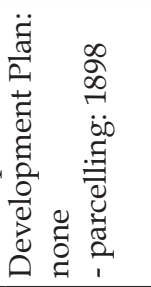 & 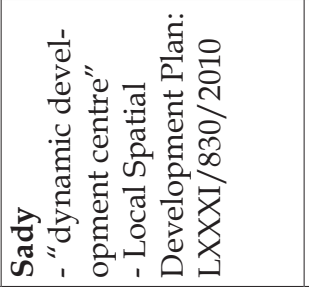 & 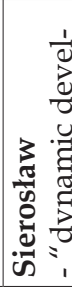 & 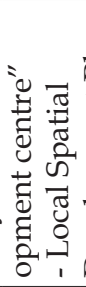 & 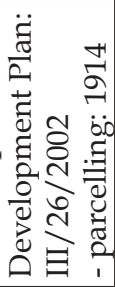 & 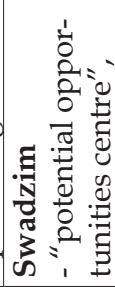 & 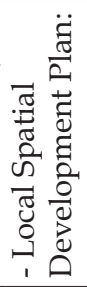 & & 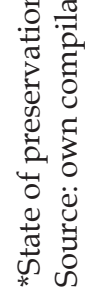 \\
\hline
\end{tabular}



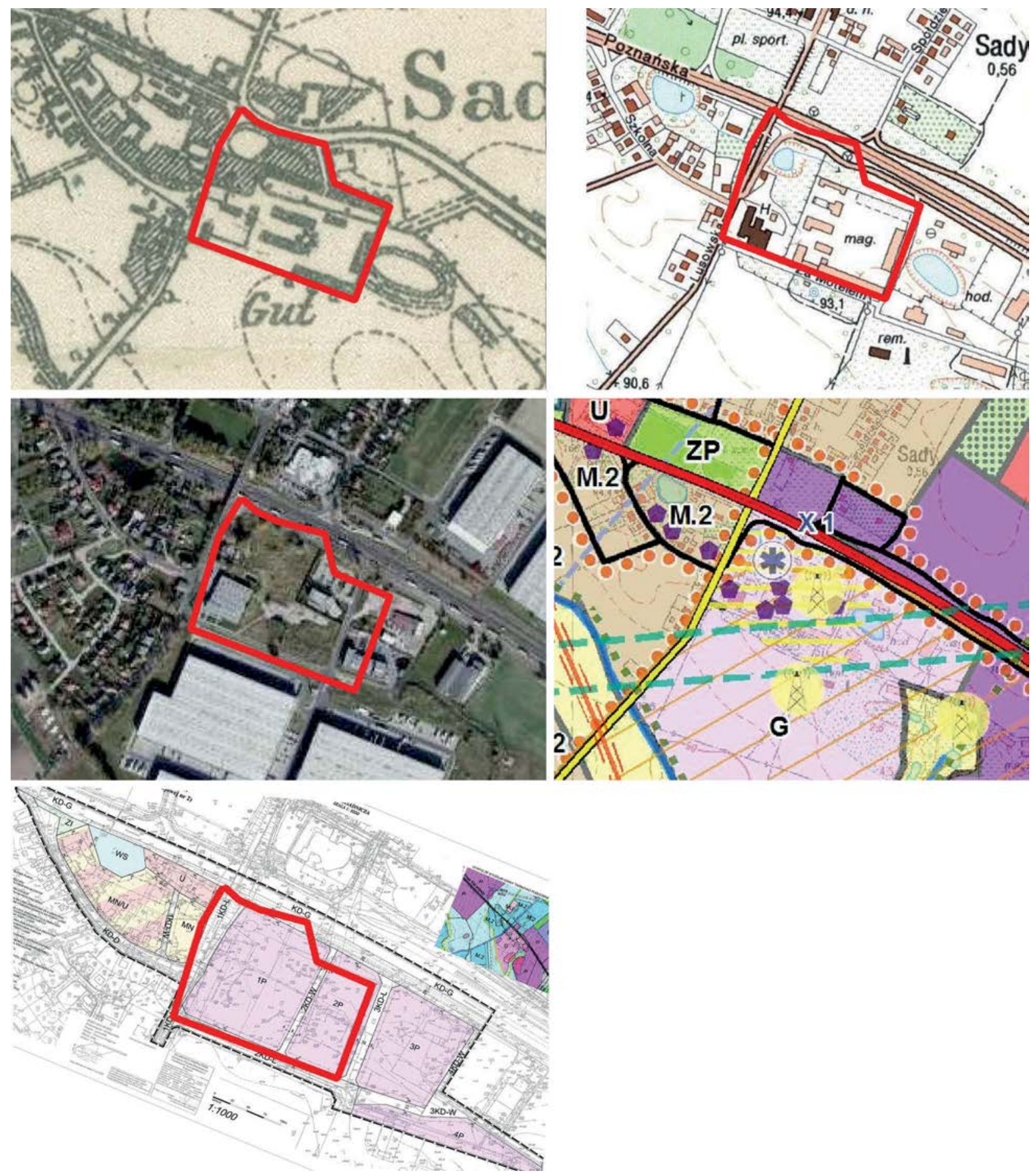

Fig. 5. Spatial arrangement of the nonexistent grange complex at Sady in the 1940s and 1970s and the present state of the complex on an orthophoto map (the range of the complex is marked with a red line), the conservation zone delineated for the complex in the Land Use Plan (yellow horizontal lines) and an extract from a drawing in the local spatial development plan.

Source: own compilation based on http://mapy.amzp.pl; http:// mapy.geoportal.gov.pl; http:// mapa.inspire-hub. pl/\#/gmina_tarnowo_podgorne. 
new buildings in the neighbourhood of those facilities and zones of conservatory protection for complexes of historical buildings (manors, palaces, parks and granges) $)^{2}$. It is forbidden to erect new buildings in historical parks and make secondary divisions in them (Tarnowo Podgórne Land Use Plan 2011, Part B). Conservation zones were delineated for 9 facilities or their fragments. In most cases the zones were delineated for manor and park complexes, in one case (the village of Sady) - for a grange complex which does not exist anymore, its area being partly occupied by contemporary buildings (Fig. 5) ${ }^{3}$.

\section{Local Spatial Development Plans for manorial and grange complexes}

Six of the facilities listed in the Land Use Plan are subject to local spatial development plans ${ }^{4}$. They are located in the villages of Tarnowo Podgórne, Ceradz Kościelny, Jankowice, Lusowo, Sady and Sierosław. There are also nine facilities not covered by legal regulations in the form of local spatial development plans. They are located in the villages of Baranowo, Sobiesiernie, Góra, Kokoszczyn (two granges), Lusówko, Przeźmierowo, Rumianek and Swadzim. In most of the plans there is no reference to the complexes under study. However, in all cases when the complexes include facilities registered as monuments, they are listed in local spatial development plans with the remark that their transformation must be consulted with conservation services. Detailed regulations concerning manorial and grange complexes can be found in the plans for Jankowice and Sierosław (Resolution No. XXII/131/2003, Resolution No. III/26/2002). The plan for Jankowice is a special planning document because it was passed in connection with the establishment of the Protected Landscape Area of the Lake Lusowo trough and the Sama River valley. Apart from recommendations concerning the protection of natural and scenic values, the plan has also regulations referring to the cultural heritage. They concern the protection

\section{Listed in the table.}

The grange complex is still marked on topographic maps issued in the late 1970s.

4 Altogether the area of former landed estates in Tarnowo Podgórne commune is covered by nine local spatial development plans. of the church, school and rectory complex in Ceradz as well as the palace and park complex, and the grange and grange workers' houses in Jankowice. The protection consists in the duty to consult a conservator about all changes planned in those areas and facilities. The second part of the plan also imposes the duty to consult conservation services about all investments in the area adjacent to the palace in Lusowo (Resolution No. XXII/132/2003). Those regulations are correct, but apparently ineffective, because the buildings in the Jankowice grange described in the plan disappeared from that place at the time when the plan was applicable. Only one granary, individually registered as a monument, remained. Apart from that, the plan sanctions the existence of single-family houses located in the area belonging to the historical park (Fig. 6). The rectory in Ceradz Kościelny, which is part of the manorial and grange complex owned by the Church, is protected by two local spatial development plans (Resolution No. LXXXI/832/2010, Resolution No. XXII/131/2003). As far as Sierosław is concerned, the plan delineates a protection zone for the park, which is listed in the Voivodeship Register of Historical Monuments. According to the plan, it is also necessary to preserve the form of the park and consult a conservator about the adaptation of the manor and barn which were part of the old grange. The plan also demands that the shop located in the park should be removed from it (Resolution No. III/26/2002).

As can be concluded from the analysis of local spatial development plans, these documents do not make use, or only minimally make use, of the possibility of regulating the rules of development of the area and handling the facilities which are remains of historical estates. Mostly they only demand that actions concerning those facilities should be consulted with an appropriate conservation office. Usually the regulations demanding consultation with conservation services refer to facilities already covered by other regulations, because they are registered as monuments (they hardly ever refer to facilities of historical value not registered as monuments). The regulations concerning the development of post-manorial parks are surprisingly scant if we consider the significance given to them by the Land Use Plan. It is worth reminding that according to the Land Use Plan, one of the goals of the spatial policy in 

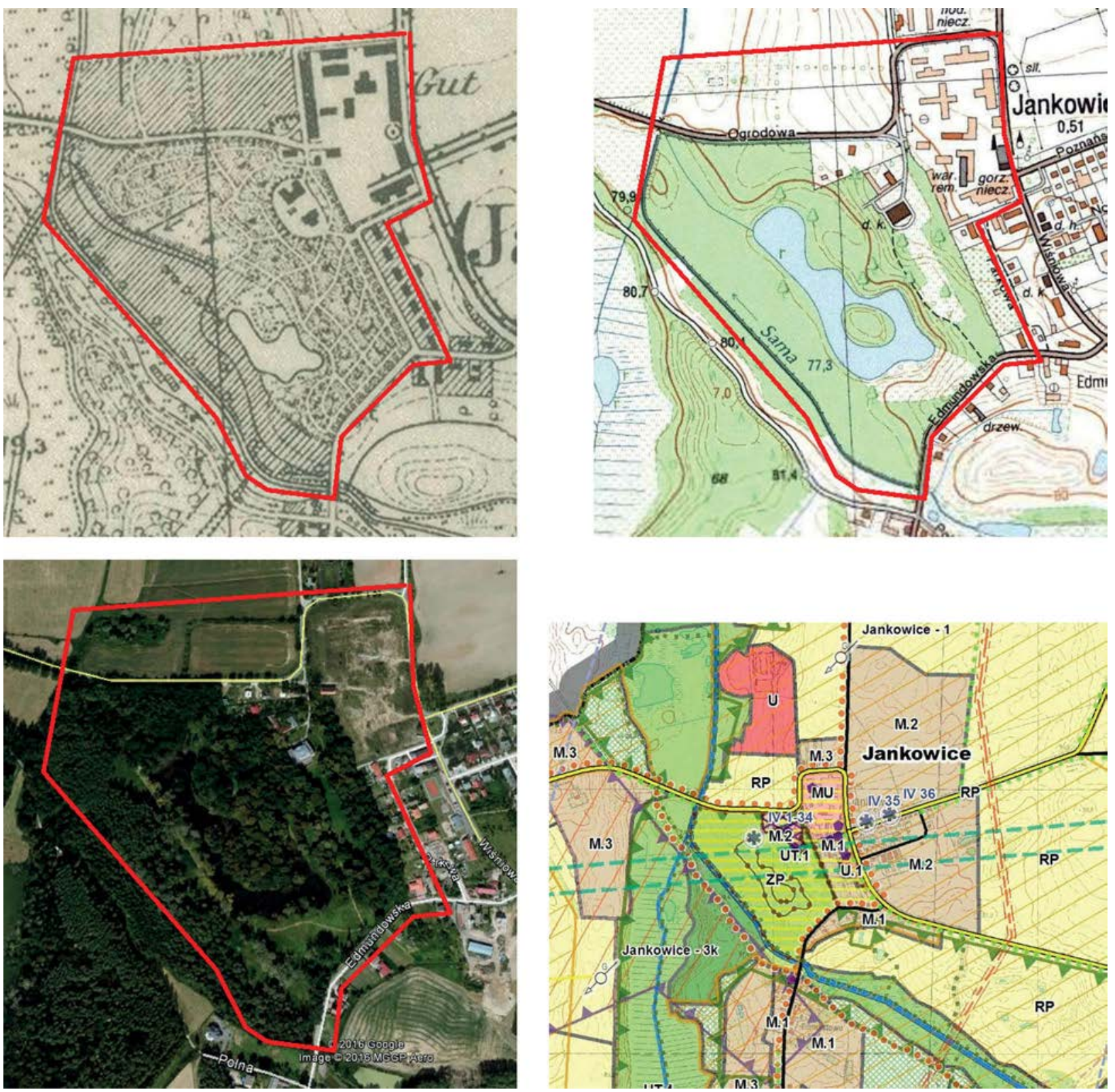

Fig. 6. Spatial arrangement of the Jankowice manorial and grange complex on maps issued in the 1940s and 1970s and the present state of the complex on an orthophoto map (the range of the complex is marked with a red line), the conservation zone delineated for the complex in the Land Use Plan (yellow horizontal lines).

Source: own compilation based on http://mapy.amzp.pl; http://mapy.geoportal.gov.pl; http://mapa.inspire-hub. $\mathrm{pl}$ /\#/gmina_tarnowo_podgorne.

the commune is to preserve and protect such facilities and to use them for the purposes of tourism and recreation.

\section{State of preservation of spatial structures} and facilities related with former estates in Tarnowo Podgórne commune and provisions of planning documents

The manorial and grange complexes in Tarnowo Podgórne commune that are the focus of this study differ in their state of preservation. The estates in Swadzim and Baranowo are the most legible as entire spatial complexes (Fig. 7). As far as the other facilities are concerned, the grange part of the estates has not been preserved at all, or only to a minimum degree. The legibility of individual estates was undoubtedly influenced by secondary ownership-related divisions that most of them had undergone. There is considerable fragmentation of ownership, and individual parts of 

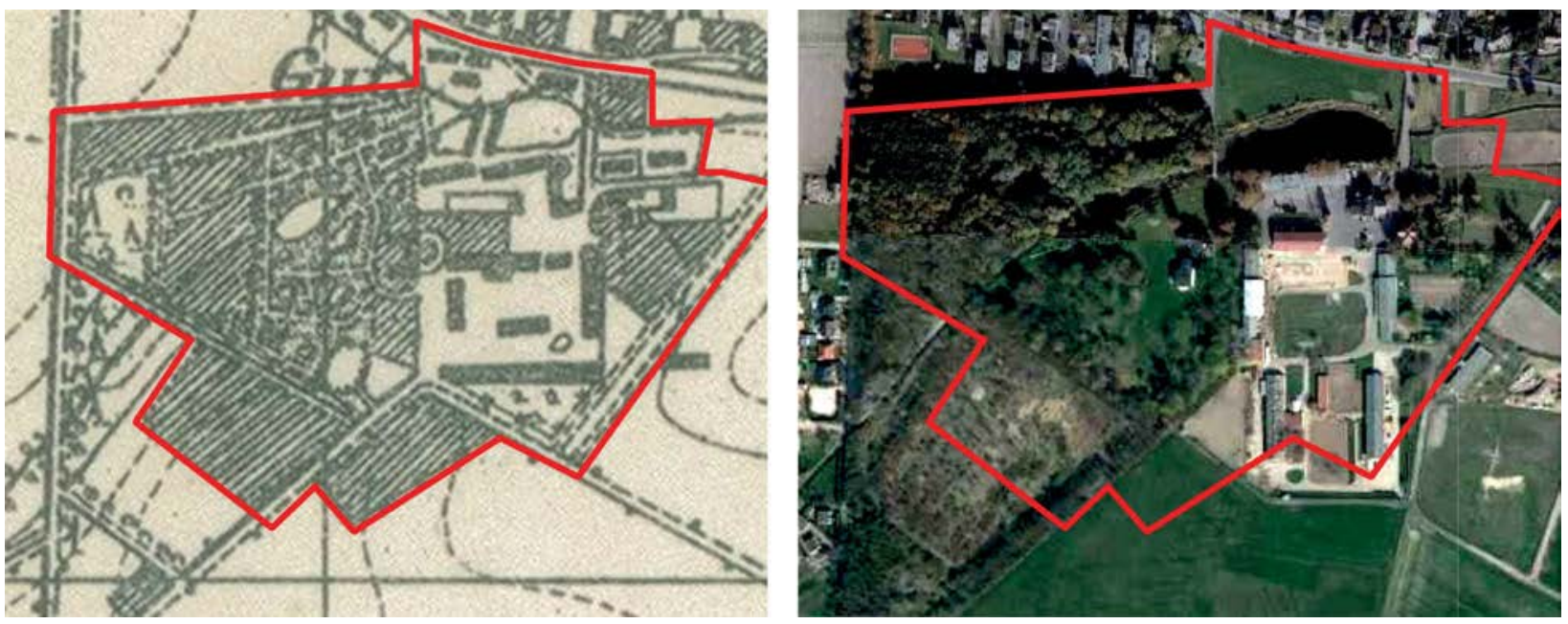

Fig. 7. Legible spatial arrangement of the manorial and grange complex in Swadzim on a map issued in the 1940s and on a contemporary orthophoto map, marked with a red line.

Source: own compilation based on http://mapy.amzp.pl; http://mapy.geoportal.gov.pl.

the complexes are administrated by different owners and in different ways. In consequence, their spatial integrity is obliterated, and it is impossible to understand their historical context and the manner in which they functioned as a whole. The estates in Tarnowo Podgórne, Góra, Sobiesiernie, Rumianek, Kokoszczyn, Lusowo, and Przeźmierowo were parcelled in the $19^{\text {th }}$ or early $20^{\text {th }}$ centuries. The parcelling of the first seven estates resulted from the intensive activity of the Prussian Settlement Commission (Königlich Preußische Ansiedlungskommission in den Provinzen Westpreußen und Posen), which purchased land estates, parcelled them, and brought German settlers to the farms thus created. Despite the activity of the Commission, residential parts of estates (a palace or manor with a park) were often preserved as wholes, more rarely, grange yards. Probably not all estates belonging to the Commission were parcelled (some of them were purchased immediately before the outbreak of World War I and there was not enough time to divide them). The fragmentation of the complexes in Sady and Lusówko was a consequence of later changes; in Przeźmierowo it was probably the consequence of a division of the grange area into building lots in the early $20^{\text {th }}$ century. In the future the facilities which still exist as wholes should be protected from secondary divisions, e.g. by adequate regulations in local spatial development plans (there are no such regulations in the documents under analysis).
Among the three groups of elements subjected to evaluation, i.e. residential buildings (palaces, manors and houses where owners or stewards resided), parks and gardens, as well as granges, green-space facilities proved to be the best preserved items. The average score for parks and gardens was 2.9 points. The state of preservation of residential buildings had a lower score, 2.8 points on average. The state of preservation of granges was definitely the worst as they scored only 1.3 points on average. The most likely cause of this situation was the overexploitation of grange estates during the communist times, loss of their functions during political changes, and poor awareness of their historical value. The fact that the post-manorial parks and gardens have been relatively well preserved may have been caused by the policy of the commune, which regarded those facilities to be particularly important for the policy of management of cultural heritage resources. The commune authorities are engaged in the maintenance of the parks in Jankowice and Lusówko as well as part of the area which used to belong to the park in Tarnowo Podgórne. They also started clean-up operations in the park in Rumianek. The park in Jankowice is the best maintained and most attractive of them since it has undergone complex clean-up operations. The fact that some parks are registered as monuments seems to influence their state of preservation. Perhaps it is not only the very fact of registration that matters, but also the fact that the registered parks are those most impressive, most valuable 

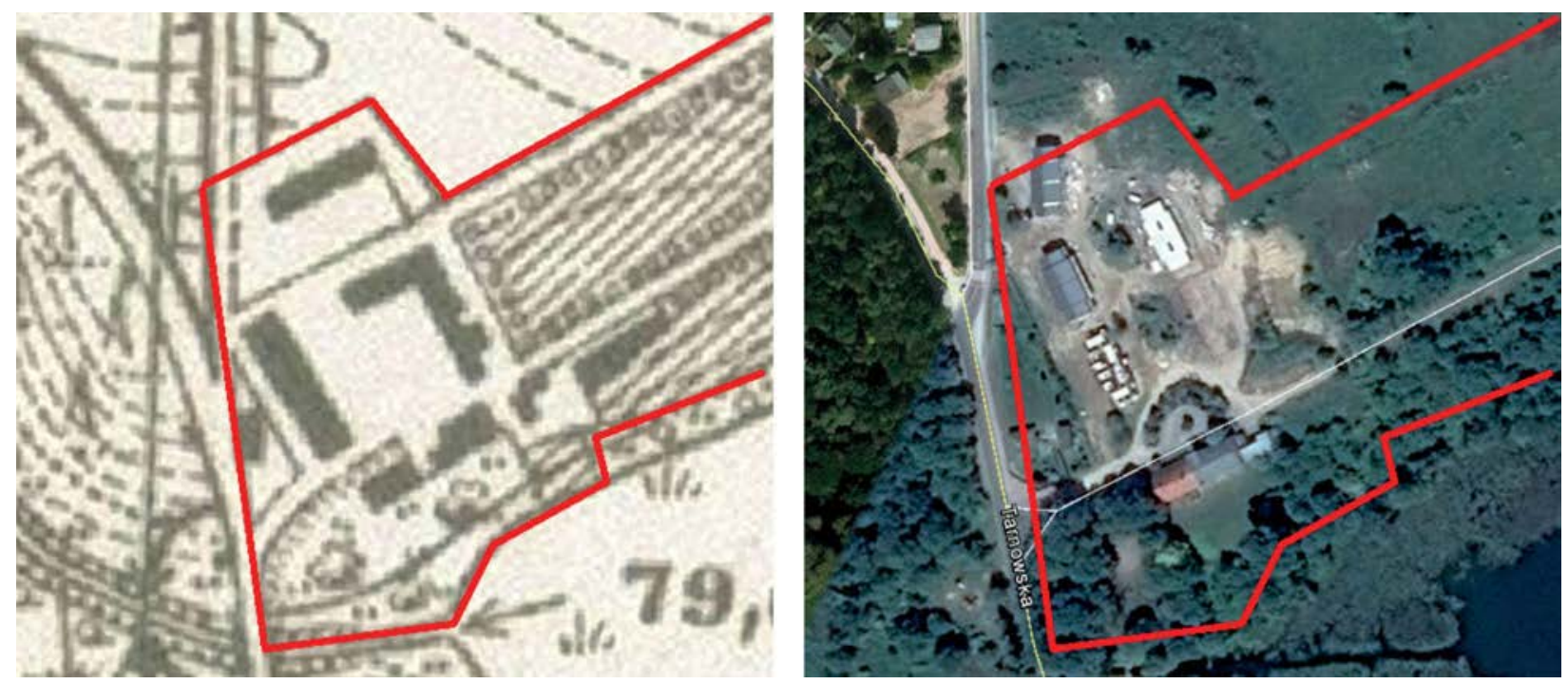

Fig. 8. Legible spatial arrangement of the manorial and grange complex in Lusówko on a map issued in the 1940s and on a contemporary orthophoto map, marked with a red line.

Source: own compilation based on http:/ / mapy.amzp.pl; http:/ / mapy.geoportal.gov.pl.

in terms of composition and historical value, and therefore present in the common awareness and believed to deserve preservation and protection. On average, the state of preservation of five facilities (Jankowice, Rumianek, Swadzim, Sierosław, Lusowo) scored 4 points. Also the state of preservation of old manors and palaces registered as monuments is slightly better than that of other facilities. On average, they scored 3.3 points. None of the granges is listed in the Voivodeship Register of Historical Monuments, an exception being the granary in Jankowice, which is the only building preserved in that grange. The commune register lists more facilities which are remains of granges, e.g. the grange barn in Sierosław, grange buildings in Swadzim and Góra, or the mill in Tarnowo Podgórne, which is probably a relic of grange buildings (Land Use Plan, p. 42). Unfortunately, some facilities listed in the commune register do not exist anymore, e.g. some grange buildings in Jankowice and the grange cowshed in Lusówko. Partial protection of manorial and grange complexes, i.e. treating their components separately and providing conservation only to individual facilities, causes a significant problem for the preservation of their historical context and the legibility of historical structures. It is important that the protection of individual facilities should be coordinated with the protection of their spatial context because interrelations between elements in space often create their real value (Tweed, Sutherland 2007). For those reasons using only lists of valuable (historical) elements when qualifying resources
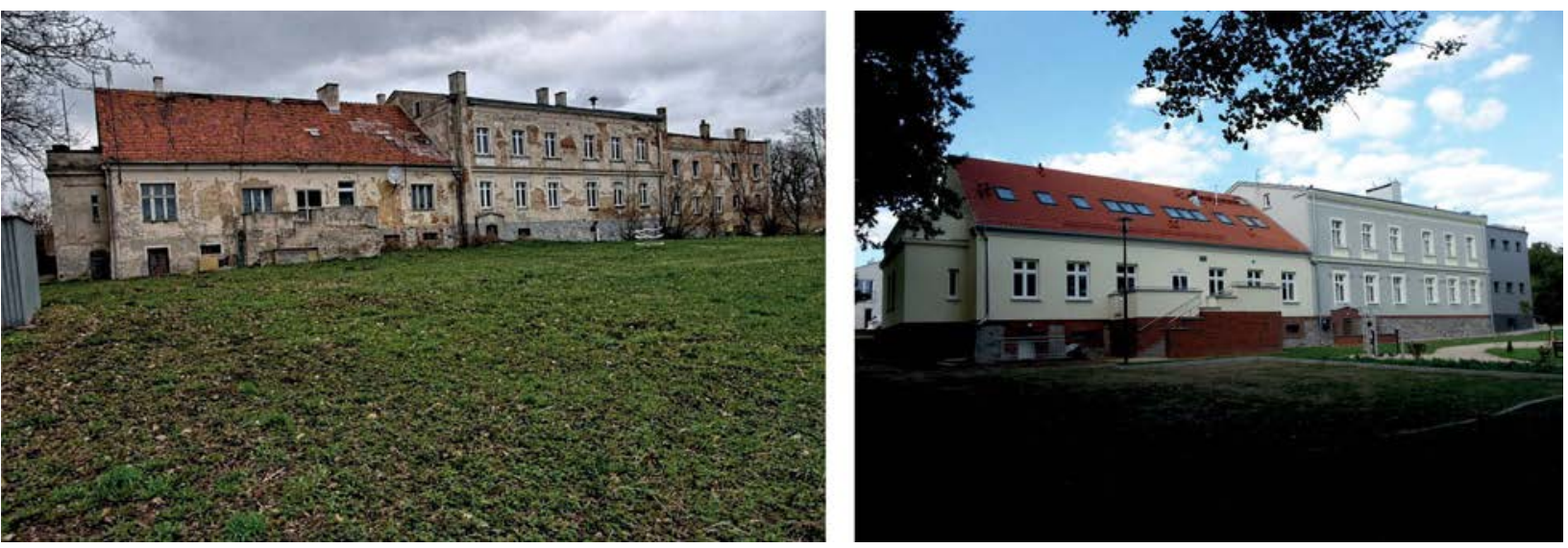

Fig. 9. Manor in Lusówko before and after revitalisation. Source: http:/ / www.polskaniezwykla.pl (photo: M. Szczepańska). 
for protection is an ineffective form of protecting the cultural heritage as a whole. However, this approach is predominant in the local spatial development plans under analysis. Nine of the complexes under study are characterised by good scenic exposition. Individual elements of a complex (structures, a mass of green space in parks, roadside alleys) significantly influence the character of a landscape, usually being its local distinguishing marks. This is particularly important for the poorly diversified agricultural landscape of Tarnowo Podgórne commune.

Besides, it should be stressed that commune authorities do not have any rural landscape studies or protection programmes for individual villages. The 2016-2020 Local Development Plan only refers to three of the facilities under study (Jankowice, Lusówko and Sierosław). According to it, there are plans to carry out revitalising operations (in the field of education and public medical services) to the amount of 14,800,000 zlotys to be spent in the next four years. The operations carried out in Lusówko deserve special attention. On the one hand, the old palace, which currently houses a school and a cultural centre, has been carefully renovated and the remains of the park have been cleaned up, but on the other, a housing estate has been built in the place of the old grange (Figs 8 and 9). As follows from the information gathered and the analyses conducted, potential protection could be given to facilities adapted for cultural and educational purposes. Also those appropriately administered should continue their current functions.

\section{Summary and conclusions}

The study revealed an ownership fragmentation of the manorial and grange complexes resulting from historical and contemporary divisions (Góra, Tarnowo Podgórne, the granges in Lusowo, Rumianek, and Sierosław). The ownership of some facilities has not been regulated (Baranowo), which causes difficulties for decisions about the use of the complex.

The authors think that the quality of plans concerning the facilities under study is unsatisfactory. The Land Use Plan includes recommendations to protect nonexistent facilities (the grange buildings in Sady, Jankowice and
Lusówko) and lists a complex located outside the commune boundaries (Chyby). Those shortcomings were probably caused by the fact that the area subject to documentation had not been examined thoroughly enough. Although the Plan acknowledges the significance of manorial and grange complexes, there are no local spatial development plans for most of them. Some local spatial development plans referring to the spatial structures under analysis are imprecise and incomplete, which results in ineffective protection. Another problem is the fact that some regulations included in local spatial development plans have not been implemented (e.g. those concerning the protection of grange buildings in Jankowice). The execution of regulations written in the local law is a wider issue. Although the Land Use Plan includes significant regulations as to how the areas surrounding historical facilities should be developed, it is striking that in most local spatial development plans they do not guarantee complete protection of spatial structures and their context. The local spatial development plans under analysis include regulations concerning the protection of individual facilities or parts of complexes, but they do not make sufficient references to relations between those parts and complexes and their surroundings.

Effective protection of manorial and grange complexes is urgently needed because they often constitute culturally, historically and scenically significant evidence of former agricultural activity in rural areas. It seems that active protection through new functions of those facilities may prevent them from being forgotten and devastated further. All development and revitalising operations should be specified after detailed spatial-functional and landscape-historical analyses that take into consideration relations between agricultural heritage resources and their surroundings.

\section{References}

Böhm A., 2006. Planowanie przestrzenne dla architektów krajobrazu. O czynniku kompozycji (Spatial planning for landscape architects. On the composition factor). Politechnika Krakowska, Kraków.

Chmielewski T., Myga-Piątek U., Solon J., 2015. Typologia aktualnych krajobrazów Polski (Typology of Poland's present-day landscapes). Przegląd Geograficzny 87 (3): 377-408. 
Chojnacka M., Wilkaniec A., 2009. Przekształcenia układów przestrzennych wsi na terenie Lednickiego Parku Krajobrazowego (Changes in rural spatial patterns in the Lednica Landscape Park). In: Kuriata Z. (ed.), Polskie krajobrazy wiejskie dawne i wspótczesne. Prace Komisji Krajobrazu Kulturowego PTG: 119-129.

Cymerman R., Falkowski J., Hopfer A., 1992. Krajobrazy wiejskie (klasyfikacje i ksztattowanie) (Rural landscapes: classifications and formation). Skrypt Akademii Rolniczo-Technicznej w Olsztynie. Wydawnictwo ART, Olsztyn.

Daugstad K., Rønningen K., Skar B., 2006. Agriculture as an upholder of cultural heritage? Conceptualisations and value judgements: A Norwegian perspective in international context. Journal of Rural Studies 22: 67-81.

Dubel K., 2003. Oceny oddziaływania na środowisko w procesie ochrony krajobrazów podmiejskich. (Assessments of environmental impact in the process of suburban landscape protection). Architektura Krajobrazu: studia i prezentacje 2003/1-2: 10-15.

Feltynowski M., 2013. Planowanie miejscowe a użytkowanie terenów w wybranych gminach wiejskich województwa łódzkiego (Local planning and land use in selected rural communes of Łódź voivodeship). Studia Obszarów Wiejskich 34: 221-231.

Głębocki B., 1998. Przemiany struktury agrarnej polskiego rolnictwa w latach 1990-1996 (Changes in the agrarian structure of Polish agriculture in the years 1990-1996). In: Głębocki B. (ed.), Przestrzenna transformacja struktury agrarnej a wielofunkcyjny rozwój wsi w Polsce. Bogucki Wydawnictwo Naukowe, Poznań: 9-74.

Gubańska R., 2008. Współczesny obraz dolnośląskiego folwarku (Contemporary image of Lower Silesian grange complexes). Prace Komisji Krajobrazu Kulturowego 10: 178-185.

Jakimowicz T., 1994. Ku pożytkowi i piękności - o zabudowie folwarków w Wielkopolsce (For use and beauty: on buildings in grange complexes in Wielkopolska). Architektura niedostrzegana. Budownictwo folwarczne Wielkopolski. Muzeum Narodowe Rolnictwa i Przemysłu Rolno-Spożywczego w Szreniawie, Wydawnictwo Abos, Poznań: 17-27.

Jaszczuk-Skolimowska B., 2008. Jakość struktur przestrzennych terenów wiejskich i matych miast w systemie planowania gminnego i lokalnego (Quality of spatial structures in rural areas and small towns in the system of communal and local planning). Doctoral dissertation prepared at the Gdańsk University of Technology, Faculty of Architecture.

Jedut R., 1998. Obiekty zabytkowe przejęte do zasobu Własności Rolnej Skarbu Państwa (Historical monuments taken over by the Agricultural Property Agency of the State Treasury). In: Głębocki B. (ed.), Przestrzenna transformacja struktury agrarnej a wielofunkcyjny rozwój wsi w Polsce. Bogucki Wydawnictwo Naukowe, Poznań: 353-411.

Kijowska J., Kijowski A., Rączkowski W., 2011. Krajobraz i polityka - wybrane aspekty wpływu decyzji politycznych na zmiany krajobrazu w Polsce (Landscape and politics: selected aspects of the effect of political decisions on landscape changes in Poland). Prace Komisji Krajobrazu Kulturowego 15: 103-115.

Kondracki J., 2000. Geografia regionalna Polski (Regional geography of Polanf). Wydawnictwo Naukowe PWN, Warsaw.

Kostrowicki A.S., 1975. Kształtowanie krajobrazu rolniczego Polski (Formation of Poland's rural landscape). In: Buch- wald K., Engelhardt W. (eds), Ksztattowanie krajobrazu a ochrona przyrody. PWN, Warsaw: 580-623.

Kowalski P., 2013. Kształtowanie wartości kulturowych i przyrodniczych szlacheckich majątków ziemskich w dawnej Polsce (Formation of cultural and natural values of landed estates in former Poland). Teka Komisji Urbanistyki i Architektury, Oddział PAN w Krakowie, 41: 141-179.

Krygowski B., 1961. Geografia fizyczna Niziny Wielkopolskiej (Physical geography of the Wielkopolska Lowland). Poznańskie Towarzystwo Przyjaciół Nauk, Poznań.

Myga-Piątek U., 2007. Kryteria i metody oceny krajobrazu kulturowego $\mathrm{w}$ procesie planowania przestrzennego na tle obowiązujących procedur prawnych (Criteria and methods of cultural landscape assessment in spatial planning in the light of legal procedures in force). Problemy Ekologii Krajobrazu 19: 101-110.

Napierała P., 2009. Ochrona krajobrazu kulturowego Sudetów Zachodnich na przykładzie gmin Mysłakowice i Bogatynia (Protection of the cultural landscape of the West Sudeten: the case of Mysłakowice and Bogatynia communes). Wiadomości Konserwatorskie 26: 13-25.

Niedźwiedzka-Filipiak I., 2009. Wyróżniki krajobrazu $i$ architektury wsi Polski południowo-zachodniej (Landscape and architectural markers of rural areas in south-western Poland). Wydawnictwo Uniwersytetu Przyrodniczego we Wrocławiu, Wrocław.

Plan Rozwoju Lokalnego 2016-2020 (2016-2020 Local Development Plan), 2015. Tarnowo Podgórne.

Raszeja E., 2002. Procedury i instrumenty ksztattowania krajobrazu na obszarach wiejskich Wielkopolski w aspekcie integracji z Unia Europejska (Procedures and instruments of landscape development in Wielkopolska rural areas in the light of the integration with the European Union). Studioteka Zarysy, Poznań.

Raszeja E., 2010. Struktura krajobrazu rolniczego w Parku Krajobrazowym im. gen. Dezyderego Chłapowskiego (Structure of the agricultural landscape in the Dezydery Chłapowski Landscape Park). Acta Scientiarum Polonorum 9(1): 97-110.

Raszeja E., Wilkaniec A., de Mezer E., 2010. Krajobraz i dziedzictwo kulturowe wsi w aglomeracji poznańskiej (Landscape and cultural heritage of the countryside in the Poznań agglomeration). Biblioteka Aglomeracji Poznańskiej No. 3, Bogucki Wydawnictwo Naukowe, Poznań.

Resolution No. III/ 26 / 2002 of the Commune Council of Tarnowo Podgórne of 17 December 2002.

Resolution No. XXII/132/2003 of the Commune Council of Tarnowo Podgórne of 4 November 2003.

Resolution No. XXII/131/2003 of the Commune Council of Tarnowo Podgórne of 4 November 2003.

Resolution No. LXXXI/832/2010 of the Commune Council of Tarnowo Podgórne of 9 November 2010.

Rylke J., Gąsowska M., 2009. Wartości krajobrazu wiejskiego i przemysłowego dla rozwoju rekreacji na przykładzie wsi warmińskich i Kanału Elbląskiego (Assets of rural and industrial landscapes for the development of recreation: an example of Warmia villages and the Elbląg Canal). Nauka, Przyroda, Technologie 3(1), 33.

Rzeszotarska-Pałka M., 2006. Tożsamość krajobrazu kulturowego wsi folwarcznych i majątków ziemskich na terenie Pomorza Zachodniego (Identity of the cultural landscape of villages of grange complexes and landed estates in West Pomerania). Przestrzeń i Forma (3): 105-116.

Rzeszotarska-Pałka M., 2016. Threats of palaces and manorsgarden ensembles on the area of West Pomerania con- 
nected with structural transformations of rural areas in 20th and 21st century. Czasopismo Techniczne 5-A/2015: 225-237.

Swensen G., Jerpasen G.B., 2008. Cultural heritage in suburban landscape planning. A case study in Southern Norway. Landscape and Urban Planning 87: 289-300.

Szczepańska M., Wilkaniec A., 2016. Przekształcenia historycznych układów przestrzennych wsi wybranych gmin województwa wielkopolskiego (Changes in historical spatial patterns of villages in selected communes of Wielkopolska). In: Heffner K., Klemens B. (eds), Obszary wiejskie - wiejska przestrzeń i ludność, aktywność społeczna i przedsiębiorczość. Studia KPZK PAN CLXVII: 123-141.

Szyda B., 2013. Urbanizacja wsi w strefie wpływów miasta a polityka przestrzenna władz gminnych. Studium miast Częstochowy, Kielc i Radomia (Urbanisation of rural areas in an urban impact zone and the spatial policy of com- mune authorities. A study of Częstochowa, Kielce and Radom). Studia Obszarów Wiejskich 34: 149-160.

Tarnowo Podgórne, Studium Uwarunkowań i Kierunków Zagospodarowania Przestrzennego (Land Use Plan), Resolution No.XII/134/2011 of the Commune Council of Tarnowo Podgórne of 21 June 2011, Part A: Analysis of spatial development conditions; Part B: Trends in spatial development.

Tweed Ch., Sutherland M., 2007. Built cultural heritage and sustainable urban development. Landscape and Urban Planning 83: 62-69.

Żarska B., 2003. Wybrane modele relacji funkcjonalnych $\mathrm{w}$ strefie styku miasta z zewnętrznymi terenami otwarty$\mathrm{mi}$ (Selected models of functional relations in the contact zone of a city and open terrain). Architektura Krajobrazu. Studia i Prezentacje 2003/1-2: 16-19. 